\title{
X-RAY TOPOGRAPHIC INVESTIGATION OF CELLULAR STRUCTURE AND ITS RELATION TO ANOTHER DEFECTS IN VARIOUS TYPES OF GaAs SINGLE-CRYSTALS
}

\author{
W. WieRzchoWSKI AND K. MAZUR \\ Institute of Electronic Materials Technology \\ Wólczyńska 133, 01-919 Warszawa, Poland
}

\begin{abstract}
The defect patterns in GaAs crystal grown using liquid encapsulated Czochralski and gradient freeze methods with various types of doping were characterized using complementary X-ray topographic methods. It was found that the cellular structure occurring in the low doped crystal is developed independently from the actual growth surface. The occurrence of the cellular structure is connected with significant lattice deformation, and some results point that significant stress can influence its formation. The high doping prevents formation of the cellular structure, but at higher doping the phenomenon of "cellular growth" can occur due to instabilities of the growth surface. The present results point that defect pattern in GaAs crystals is more affected by the type of doping than by the choice of the growth method.
\end{abstract}

PACS numbers: $61.10 .-\mathrm{i}$

\section{Introduction}

The important defect, described in many papers concerning the structural investigation of GaAs crystal is the cellular structure of dislocations [1-6]. The cellular structure is believed to be the result of dislocation climbing and formation of precipitates under the influence of thermal stress in the material containing high concentration of interstitial atoms. To our best knowledge the existing papers do not discuss the mechanism of its formation in detail.

In the present work the cellular structure and other defects in different types of GaAs crystals are studied with complementary X-ray topographic methods. 


\section{Experimental}

Most of the investigated crystals were obtained in the Semiconductor Compounds Department of Institute of Electronic Materials Technology (ITME) and the samples were delivered to us due to courtesy of Dr. A. Hruban. Both liquid encapsulated Czochralski (LEC)-grown crystals and horizontal gradient freeze (GF)-grown crystals were examined. The LEC crystals were mostly of semi-insulating (SI) type either low doped with dopant concentration smaller than $10^{17}$ atoms $/ \mathrm{cm}^{3}$ or isoelectronically indium doped with concentration $1-2 \%$. The GF-grown crystals were silicon doped in the concentration $10^{18}-10^{19}$ atoms $/ \mathrm{cm}^{3}$ and some of them doped both with silicon and with up to $2 \%$ indium. At high concentration of silicon or indium the motion and multiplication of dislocations was decreased.

The samples were usually cut perpendicular or obliquely to the growth axis. A small number of samples was cut along the growth axis and were important for studying of the development of defects during growth process and their relation to the growth surface.

We used three complementary X-ray topographic methods:

1. the Lang transmission method,

2. the Berg-Barrett method (with elimination of $\mathrm{Cu} K_{\alpha_{2}}$ line),

3. the double-crystal method.

The Lang transmission topographs were exposed in $\mathrm{Cu} K_{\alpha_{1}}$ radiation with dynamical contrast. Reasonable exposure times were obtained in symmetrical 220, 400 and 422 reflections where anomalous transmission is high.

The Berg-Barrett method can easily reveal the groups of dislocations in GaAs, but its ability to reveal any undecorated dislocation is not quite obvious but it was convenient to study the cellular structure and mosaic blocks.

In view of short exposure times we employed double-crystal method for initial evaluation of the sample perfection. The method was also used for studying the lattice deformation making a series of topographs at the azimuths differing by $180^{\circ}$. In the material with lower dislocation densities it enabled the identification of dislocations type and Burgers vector sense using the numerical simulations. The numerical program taking into account the finite divergence of the beam in double-crystal experiment and providing a possibility of large area simulations was revealed [7].

\section{Results and discussion}

The cellular structure of dislocations was observed in all investigated low doped SI LEC crystals apart from the samples cut from the initial parts where the structure was not developed. The crystals with developed cellular structure exhibit high lattice deformation. It may be illustrated by equal orientation contours, similar to those discussed in [8], on a representative sample shown in Fig. 1. The contours indicate that most of the deformation consist of lattice bending. In some regions we indicated the changes in interplanar spacings $\Delta a / a$ at the level $10^{-5}$. 

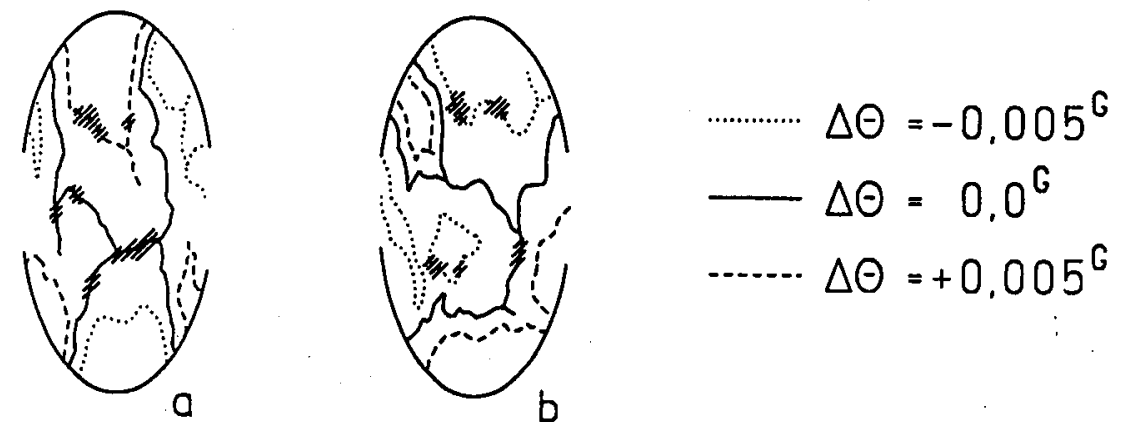

Fig. 1. Equal orientation maps in two perpendicular planes of a representative low doped LEC SI crystal containing cellular structure, drawn on the base of series of $400_{G a A s},-400_{G a A s}$ double-crystal topographs. The regions with interplanar spacing changes are marked.

In view of the experiments by Nakajima et al. [9] with lattice measurement in diced samples it may be expected that the interplanar spacing is almost entirely due to elastic strain. It was also found that the changes of interplanar spacings occurred in the region where the cellular structure is more dense.

From the double-crystal topograph we established that the misorientation was randomly distributed between grains containing number of cells. It is usually of the order of less than $0.1^{\prime \prime}$ between cells and few seconds between the groups. An extreme case was found in a bad quality crystal where the blocks grouping the cellular structure have the misorientation of single degrees. Our former investigation with double-crystal and plane polariscope method [10] pointed that the residual stress distribution is of dominant radial character and is more regular than the deformation pattern.

It is not easy to distinguish the contribution to the topographic contrast due to the dislocation and small arsenic inclusions [11,12]. The existence of these two kinds of defects in the cell wall was established in the number of papers, especially by Chabli et al. [6]. It is to some extent confirmed in the investigation of Pawlowska [13] by means of cathodoluminescence topography. Some arguments were found from the double-crystal topographs of the large cells.

The dislocation contrast was studied by us experimentally in heavy Si-doped crystals where the dislocation density was locally $10^{2} / \mathrm{cm}^{2}$ and theoretically by computer simulations. It was established that double-crystal images of dislocations in GaAs are dominated by a characteristic "rosette" roughly corresponding to the deformation field. It is reversible with the change of the Burgers vector sense and with the change of the peak side. It also undergoes characteristic changes in equivalent reflections or changing the azimuth in symmetrical reflections. The images of small inclusions, especially situated at certain depth will behave in different way. Firstly, it will not form the "rosette" with sectors coming from a 
single point. Secondly, the deformation field of small inclusion has approximately circular symmetry and its image should not change in equivalent reflections. Some elements forming the cells contrast in Fig. 2 can to some extent be classified in these two groups.

The topographs of the longitudinal section of the low vanadium doped crystal is shown in Fig. 3. It provides a very important result that the cells are not connected with the growth surface. No elongation of cells along the growth axis was also observed.

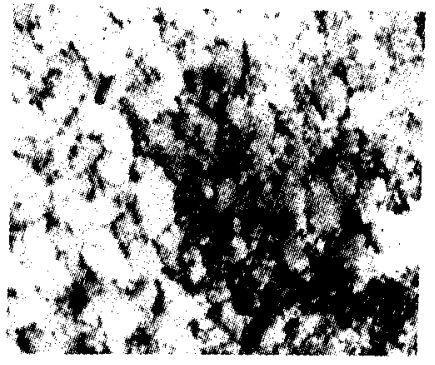

Fig. 2

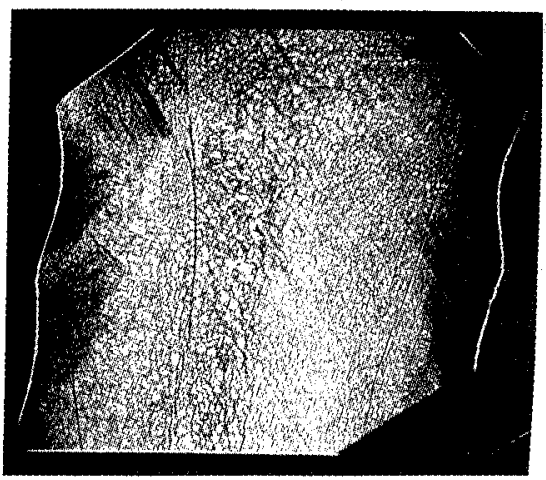

Fig. 3

Fig. 2. The enlarged part of the $511_{G e},-511_{G a A s}$ topograph (photographic positive) of LEC crystal with the cellular structure

Fig. 3. The $220 \mathrm{Cu} K_{\alpha_{1}}$ Lang topograph of a sample cut along the growth axis of a SI LEC crystal pointing that the cellular structure exhibits no elongation along the growth axis and no connection with the growth surface.

The cellular structure was observed in some cases also in the silicon doped GF crystals. We observe dense cellular structure in some regions of these crystals, while most of the crystal volume contained low or very low dislocation density. Actually the cellular structure occurred close to the defects producing extended deformation field, e.g. large inclusion seen. Some of the defect pattern were similar to those shown in [4].

The different phenomenon, accidentally similarly named is the structure due to the cellular growth. These phenomenon in heavily doped GaAs was described by Schumann et al. [14], and recently observed by Pichaud et al. [15].

The cellular growth is due to the growth surface instabilities caused by constitutional supercooling. In our case it was observed in the indium doped crystal with highest indium concentration both LEC and double silicon and indium doped GF crystals. The cellular growth occurred in the lower parts of crystals with high indium concentration. That behavior is in accordance with the theory formulated by Hurle [16].

The cells produce characteristic "envelope" pattern due to growth on four $\{111\}$ facets in each cell, shown in Fig. 4. It is characteristic that cells are extended 


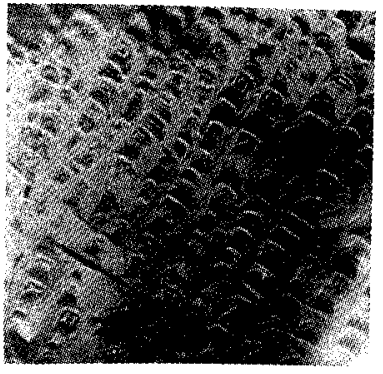

Fig. 4

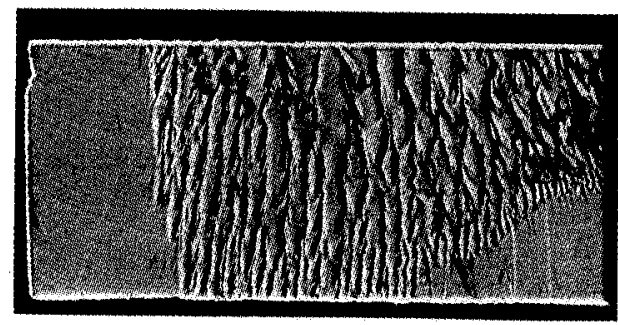

Fig. 5

Fig. 4. The enlarged fragment of the double-crystal image of silicon and indium doped GF GaAs crystal with structure due to cellular growth, exhibiting "envelope" pattern due to $\{111\}$ facets

Fig. 5. The Lang topograph of the sample cut from GF crystal silicon and indium doped along the growth axis. It may be seen that the cells are very long. The growth direction points down.

very long as is seen in the topograph of the sample cut along the growth axis from GF crystals (see Fig. 5). In the LEC crystals the cells were isolated from each other but in the GF they were neighboring and then grains formed a structure to some extent similar to the shape of the previously considered cellular structure.

It is characteristic that heavily indium doped crystals contained high areas with low dislocation density. In the indium doped LEC crystals we observed dense grid of slip bands in the outer zone of the crystal. The slip bands occurred sometimes, but with much lower density in low doped LEC crystals and practically were not present in GF crystals. These results point to the role of the cellular structure in the stress relaxation and to the lower thermal stress during the growth of presently investigated GF crystals.

\section{Conclusions}

The samples of the crystals characteristic of the present applications of GaAs were studied and the defects most characteristic of these crystal are considered. We found some characteristic features of cellular structure as e.g.: occurrence of misorientation between grains containing some cells and formation of cellular structure under the influence of long-range deformation field even when high doping was introduced. We compared observations of the cellular structure and the cells due to the cellular growth. The comparison of crystals grown with different methods points that the defect pattern is related to the doping type rather than to the method of growth. 


\section{References}

[1] T. Sekiguchi, K. Sumino, Jpn. J. Appl. Phys. 26, L179 (1987).

[2] T. Kamejima, F. Shimura, Y. Matsumoto, H. Watanabe, J. Matsui, Jpn. J. Appl. Phys. 21, L721 (1982).

[3] A. Chabli, E. Molva, A. George, F. Bertin, P. Bunod, J. Bletry, in: E-MRS, Strasbourg June 1986, p. 27.

[4] A. Grant-Elliot, A. Flat, D.A. Vanderwater, J. Cryst. Growth 121, 349 (1992).

[5] F. Ponce, Inst. Phys. Conf. Ser. 76, 1 (1985).

[6] D. Korytár, V. Daniška, I. Priecel, M. Babinský, J. Cryst. Growth 121, 440 (1992).

[7] W. Wierzchowski, Proc. 15th Conference on Applied Crystallography, August 9-12, 1992, Cieszyn, Abstracts, p. 31. in press.

[8] T. Ishikawa, T. Kitano, J. Matsui, Jpn. J. Appl. Phys 24, 968 (1985).

[9] M. Nakajima, T. Sato, T. Inada, T. Fukuda, K. Ishida, Appl. Phys. Lett. 49, 1251 (1986).

[10] G. Adamkiewicz, A. Bajor, W. Wierzchowski, Cryst. Res. Technol. 23, 901 (1988).

[11] T. Kitano, T. Ishikawa, J. Matsui, Phys. Status Solidi A 115, 383 (1989).

[12] E. Zieliniska-Rohozińska, J. Cryst. Growth 87, 154 (1988).

[13] M. Pawlowska, I. Dąbrowska, W. Orłowski, Materiaty Elektroniczne (Poland) 63, 11 (1988).

[14] M. Schumann, Krist. Technik 11, 41 (1976).

[15] B. Pichaud, F. Minari, B. Billia, J. Cryst. Growth 80, 469 (1987).

[16] D.T.J. Hurle, Solid State Electron. 3, 37 (1961). 